\title{
BMJ Open Association of the vitamin D binding protein polymorphisms with the risk of type 2 diabetes mellitus: a meta-analysis
}

\author{
Gaoshuai Wang, ${ }^{1}$ Yuqian Li, ${ }^{2}$ Linlin Li, ${ }^{1}$ Fei Yu, ${ }^{1}$ Lingling Cui, ${ }^{1}$ Yue Ba, ${ }^{1}$ Wenjie Li, ${ }^{1}$ \\ Chongjian Wang ${ }^{1}$
}

To cite: Wang G, Li Y, Li L, et al. Association of the vitamin $\mathrm{D}$ binding protein polymorphisms with the risk of type 2 diabetes mellitus: a meta-analysis. BMJ Open 2014:4: 005617. doi:10.1136/bmjopen-2014005617

- Prepublication history for this paper is available online. To view these files please visit the journal online (http://dx.doi.org/10.1136/ bmjopen-2014-005617).

GW and YL contributed equally.

Received 3 May 2014 Revised 28 July 2014 Accepted 30 July 2014

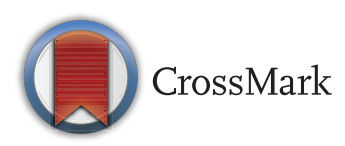

${ }^{1}$ Department of Epidemiology and Biostatistics, College of Public Health, Zhengzhou University, Zhengzhou, Henan, People's Republic of China

${ }^{2}$ Department of Clinical Pharmacology, School of Pharmaceutical Science, Zhengzhou University, Zhengzhou, Henan, People's Republic of China

Correspondence to Dr Chongjian Wang; tjwcj2005@126.com and Dr Wenjie Li; Iwj@zzu.edu.cn

\section{ABSTRACT}

Objective: Previous studies on the association between vitamin $\mathrm{D}$ binding protein $(D B P)$ polymorphisms and the risk of type 2 diabetes mellitus (T2DM) have produced conflicting results. The purpose of this meta-analysis was to examine whether $D B P$ polymorphisms are associated with the risk of T2DM.

Design: Systematic review and meta-analysis. Methods: All eligible studies were searched and acquired from the Cochrane, Pubmed, ISI, CNKI (Chinese) and Wanfang (Chinese) databases. ORs with corresponding $95 \%$ Cls were computed to estimate the association between DBP polymorphisms and T2DM. In addition, heterogeneity test, meta-regression and sensitivity analysis were also conducted.

Results: Six studies, which included 1191 cases and 882 controls, met the inclusion criteria and were included in the meta-analysis. The results showed that no significant associations were found between codon 416 and codon 420 polymorphisms in the DBP and the risk of T2DM in the overall analyses. In stratified analysis, significant associations between the codon 420 polymorphism and T2DM were found in Asians (allele Lys vs Thr: OR (95\% Cl) 1.49 (1.19 to 1.85), genotype Lys/Thr versus Thr/Thr: OR ( $95 \% \mathrm{Cl}) 1.80$ (1.36 to 2.38), and Lys/Thr+Lys/Lys versus Thr/Thr: OR $(95 \% \mathrm{Cl}) 1.81$ (1.37 to 2.39$)$, respectively) but not in Caucasians. For the codon 416, the significant association with T2DM was also detected in Asians (genotype Glu/Asp+Glu/Glu vs Asp/Asp: OR (95\% Cl) 1.36 (1.04 to 1.78)) but not in Caucasians.

Conclusions: This meta-analysis demonstrated that the $D B P$ polymorphism was moderately associated with increased susceptibility to T2DM in Asians, but a similar association was not found in Caucasians. It suggested that ethnicity might be the potential factor associated with heterogeneity.

\section{INTRODUCTION}

Type 2 diabetes mellitus (T2DM) is a complex metabolic disorder caused by the interaction of multiple genetic and environmental factors, and the suggested overall genetic contribution is around $50-70 \%$; until

\section{Strengths and limitations of this study}

- This is the first systematic review and meta-analysis to evaluate the association between vitamin $D$ binding protein $(D B P)$ polymorphisms and type 2 diabetes mellitus (T2DM).

- All the articles included in this manuscript must confirm that all controls were in the Hardy-Weinberg equilibrium. Heterogeneity test, meta-regression and sensitivity analysis were also conducted.

- It still needs to be demonstrated that $D B P$ polymorphisms were associated with T2DM in other places such as Africa and South America.

now, more than 60 genes have been reported to relate to $\mathrm{T} 2 \mathrm{DM}$, and these genes always contain polymorphisms that modify their function. ${ }^{1-3}$

Vitamin D binding protein $(D B P)$, also known as a group-specific component protein $(G c)$, is a multifunctional serum glycoprotein. ${ }^{4}$ As a major plasma carrier protein of vitamin D sterols, $D B P$ is essential for the intracellular metabolism of vitamin $\mathrm{D} .{ }^{5}$ So variations in $D B P$ might influence the amount and activity of vitamin $\mathrm{D}$, and then alter insulin secretion, $\beta$-cell dysfunction and glucose metabolism. ${ }^{6-8}$

DBP gene spans 35 kilobase pairs and contains 13 exons and 12 introns, and maps to the long arm of chromosome 4 (4q12-q13). ${ }^{5} 9$ There are two major polymorphisms in DBP which were studied. A nucleotide exchange ( $G A T$ to $G A G$ ) in position 416 contributes to an Asp to Glu exchange. Additionally, the $A C G$ to $A A G$ in position 420 changes Thr to Lys. ${ }^{10} \mathrm{~A}$ few previous studies have been carried out to access the association between DBP polymorphisms and risk of T2DM in different populations; however, the results are inconsistent and inconclusive. ${ }^{11-16}$ Therefore, it remains uncertain if DBP polymorphisms are really associated with an increased risk of T2DM. The purpose of this study was to assess the association of DBP polymorphisms with 
T2DM by conducting a systematic review and meta-analysis from individual data sets of all relevant studies published to date.

\section{MATERIALS AND METHODS}

\section{Literature and search strategy}

The study was performed according to the Preferred Reporting Items for Systematic Reviews and Meta-Analysis (PRISMA) statement. ${ }^{17}$ A computerised literature search was conducted using the Cochrane, Pubmed, ISI, CNKI (Chinese) and Wanfang (Chinese) databases for relevant articles published in English and Chinese before the end of March 2014. The search terms were as follows: "vitamin D binding protein or groupspecific component protein (DBP or Gc)" and "polymorphism or variant" and "type 2 diabetes mellitus (T2DM)". In addition, the reference lists of original and review articles were also researched to identify any additional relevant articles using the previous databases.

The included studies must meet the following criteria: (1) the design had to be a case-control or correlation study; (2) there is a description of DBP genotype frequencies in cases and controls provided; (3) the study evaluated the association between DBP polymorphisms and T2DM; (4) there were sufficient data for estimating an OR with $95 \%$ CI. In all the studies, genomic DNA from people was extracted from blood and DBP status was determined by analysis of the gene through PCR single strand conformation polymorphism (PCR-SSCP), PCR restriction fragment length polymorphisms (PCR-RFLP), PCR-based denaturing highperformance liquid chromatography (DHPLC), conforming two-pair primers (CTPP) or biochip.

\section{Data extraction}

Data were extracted and entered into a database by two investigators (GW and YL) independently. For conflicting evaluations, an agreement was reached following a discussion. The data extracted were as follows: the surname of the first author, journal, date of publication, country of origin, ethnicity, study design, characteristics of cases and controls, matching variables, mean age of the case group, number of cases and controls, genotyping method, genotype distribution of cases and controls, quality control for genotyping assay and the Hardy-Weinberg equilibrium (HWE). In addition, the studies were appraised by the publication influence factor, restriction of control, data completion and so on.

\section{Statistical analysis}

OR with $95 \%$ CI was calculated to estimate the relationship between the DBP polymorphisms and T2DM. The Newcastle-Ottawa Scale (NOS) was used to assess the quality of studies on three broad perspectives. ${ }^{18} \mathrm{~A}$ 'star system' (out of 9 stars) was used to describe the quality of studies. A random-effects model would first be used to estimate the pooled OR value, and then a fixed-effects model would be applied when $\mathrm{p}>0.10$ in the heterogeneity analysis. We assessed heterogeneity in effect estimates using $\mathrm{I}^{2}$, $\mathrm{p}$ value for heterogeneity $(\mathrm{Ph})$. If there is a significant heterogeneity $(\mathrm{Ph} \leq 0.1$ or $\mathrm{I}^{2}>50 \%$ ) among studies, we will explore the source of heterogeneity by meta-regression and perform sensitivity analysis. Sensitivity analysis was performed by removing the lowest quality of study which is inconsistent with HWE to assess the stability of the results. For each polymorphism site, four genetic models were used (using codon 416 as an example): allele comparison (Glu vs Asp), codominant model (Glu/Glu vs Asp/Asp, Glu/ Asp vs Asp/Asp), dominant model (Glu/Asp+Glu/Glu vs Asp/Asp). Studies were stratified according to source of cases, ethnicity and source of controls for exploration of heterogeneity. All analyses were performed by the STATA software package, V.11.0 (STATACorp., College Station, Texas, USA).

\section{RESULTS}

\section{Characteristics of included studies}

The detailed selection process is shown in figure 1. In total, 92 studies were identified using five electronic databases and a manual search. Fourteen articles were excluded for duplicate publication. After reading the title and abstract, fifty-nine reports were excluded (48 articles were not relevant to DBP polymorphism, 5 articles were not relevant to T2DM, and 6 articles were review articles). After the full-text evaluation, 13 articles were excluded with reasons for no available data $(n=6)$, duplicate publications $(n=4)$, no case-control studies $(n=2)$, and no human study $(n=1)$. Finally, six studies ( 4 in English and 2 in Chinese) were included in this meta-analysis.

The characteristics of these studies are listed in table 1. There were three studies based on the Asian population and three studies conducted on the Caucasian population. These six articles were all about codon 416 and codon 420, and included 1191 cases and 882 controls. The detection methods of genotype were all PCR-RFLP. Except for one study, ${ }^{11}$ the genotype distributions in the controls of all studies were inconsistent with HWE. The quality of studies was described by the number of stars.

\section{Quantitative data synthesis}

Figures 2 and 3 summarise the ORs with corresponding 95\% CIs for the association between codon 416 and codon 420 polymorphisms in the DBP and the risk for T2DM under all genetic models. Overall, there was no evidence of an association between codon 416 and codon 420 polymorphisms and the risk of T2DM when all the eligible publications were pooled into the meta-analysis. In the subgroup analysis by ethnicity, a significant association between the codon 420 polymorphism and the risk of T2DM was found in Asians (allele Lys vs Thr: OR (95\% CI) 1.49 (1.19 to 1.85), genotype Lys/Thr versus Thr/Thr: OR (95\% CI) 1.80 (1.36 to 2.38), and Lys/Thr+Lys/Lys versus Thr/Thr: OR (95\% 
Figure 1 Flow diagram for literature inclusion and exclusion (DBP, vitamin D binding protein; T2DM, type 2 diabetes mellitus).

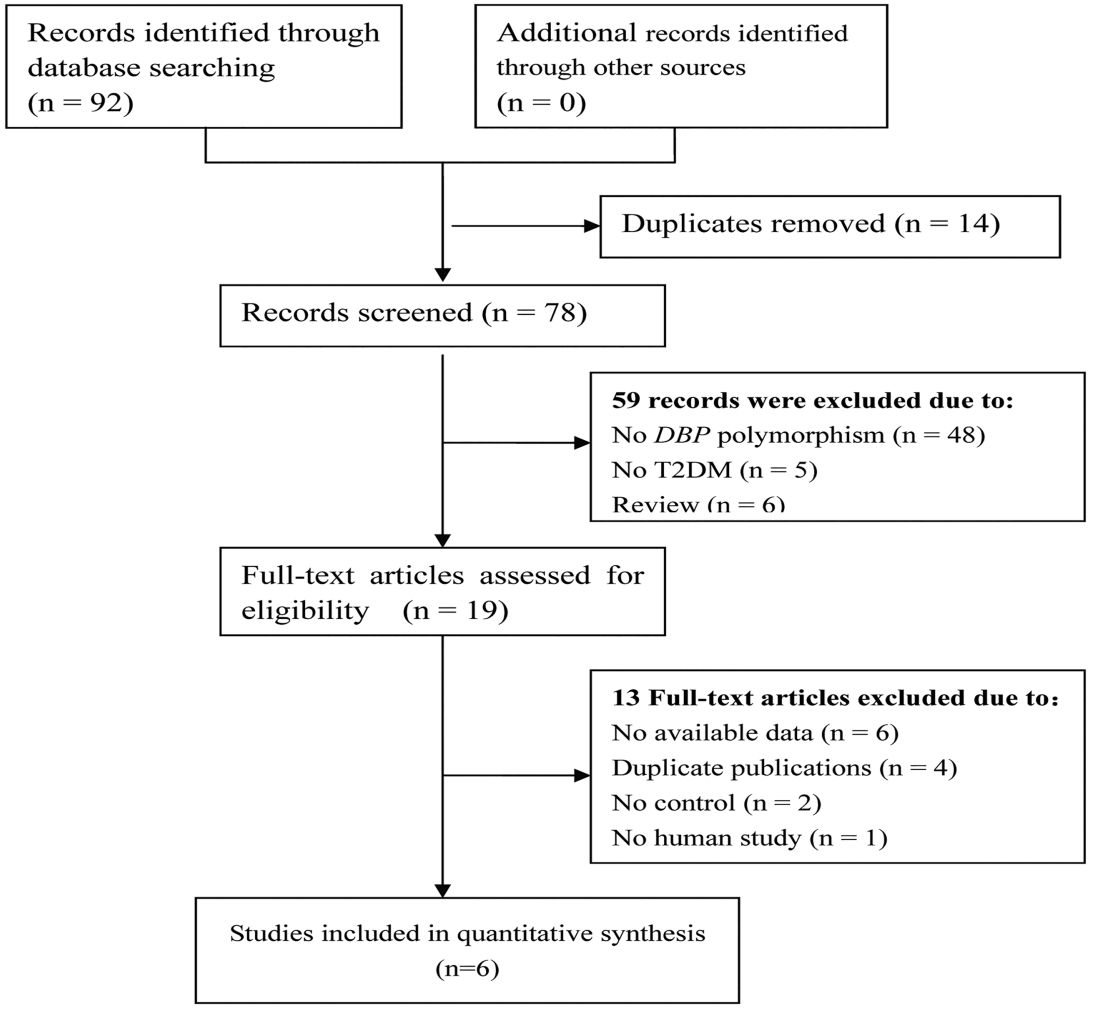

CI) 1.81 (1.37 to 2.39), respectively). For codon 416, a similar association was also detected in Asians (Glu/Asp + Glu/Glu vs Asp/Asp: OR (95\% CI) 1.36 (1.04 to 1.78). For Caucasians, no significant associations between codon 416 and codon 420 polymorphisms and T2DM were observed under all the four genetic models. The results are presented in tables 3 and 4 .

\section{Heterogeneity test}

Heterogeneity analyses of the four genetic models were conducted respectively. The results showed that there was significant heterogeneity among the six studies. Then the source of heterogeneity was explored. Studies were stratified by the following characteristics: source of cases, ethnicity and source of controls. Results of meta-regression showed that the systemic results were not affected by these characteristics except for ethnicity $(\mathrm{p}<0.05)$ in the codon 420 polymorphism (shown in table 2). In codon 420, under the dominant model, the overall $\mathrm{I}^{2}$ is $66 \%(\mathrm{Ph}=0.01)$, but in the analysis of ethnicity the heterogeneity was significantly removed in Asians $\left(I^{2}=14 \%, P h=0.31\right)$ and in Caucasians $\left(I^{2}=0 \%\right.$, $\mathrm{Ph}=0.52)$. Similar results were also detected in the allele model. Results are shown in tables 2-4.

\section{Sensitivity analysis}

To further strengthen our conclusions, sensitivity analysis was performed by removing the lowest quality of study ${ }^{11}$ not in HWE. For the overall analysis, the OR and $95 \%$ CI were similar with the former OR and 95\% CI when the study was omitted. For the Asian subgroup analysis, when the study was omitted, the OR of the dominant model of codon 416 , which was significant previously ( $\mathrm{OR}=1.36,95 \%$ CI 1.04 to 1.78 ), became insignificant $(\mathrm{OR}=1.10,95 \%$ CI 0.76 to 1.58$)$. Results are presented at tables 3 and 4 .

Table 1 The basic characteristics of the included studies

\begin{tabular}{|c|c|c|c|c|c|c|c|}
\hline \multirow[b]{2}{*}{$\begin{array}{l}\text { First author } \\
\text { (year) }\end{array}$} & \multirow[b]{2}{*}{ Country (ethnicity) } & \multicolumn{2}{|c|}{ Participants } & \multirow[b]{2}{*}{$\begin{array}{l}\text { Cases } \\
\text { source }\end{array}$} & \multirow{2}{*}{$\begin{array}{l}\text { Cases age } \\
\text { (mean } \pm S D, \\
\text { year) }\end{array}$} & \multicolumn{2}{|c|}{ HWE (p value*) } \\
\hline & & Cases & Controls & & & $\begin{array}{l}\text { Codon } \\
420 \\
\end{array}$ & $\begin{array}{l}\text { Codon } \\
416\end{array}$ \\
\hline Ye WZ (2001) & France (Caucasian) & 237 & 143 & Hospital & $63.00 \pm 11.00$ & 0.730 & 0.710 \\
\hline Klupa T (1999) & America (Caucasian) & 215 & 163 & Population & $62.30 \pm 5.40$ & 0.841 & 0.811 \\
\hline Malecki MT (2002) & Poland (Caucasian) & 231 & 162 & Population & $57.10 \pm 11.40$ & 0.148 & 0.081 \\
\hline Shao XJ (2012) & China (Asian) & 105 & 105 & Hospital & $60.67 \pm 11.69$ & 0.223 & 1.000 \\
\hline Ren W (2003) & China (Asian) & 201 & 110 & Population & $52.65 \pm 12.07$ & 0.372 & 0.846 \\
\hline Hirai M (1998) & Japan (Asian) & 202 & 199 & Population & NR & 0.0001 & 0.025 \\
\hline
\end{tabular}




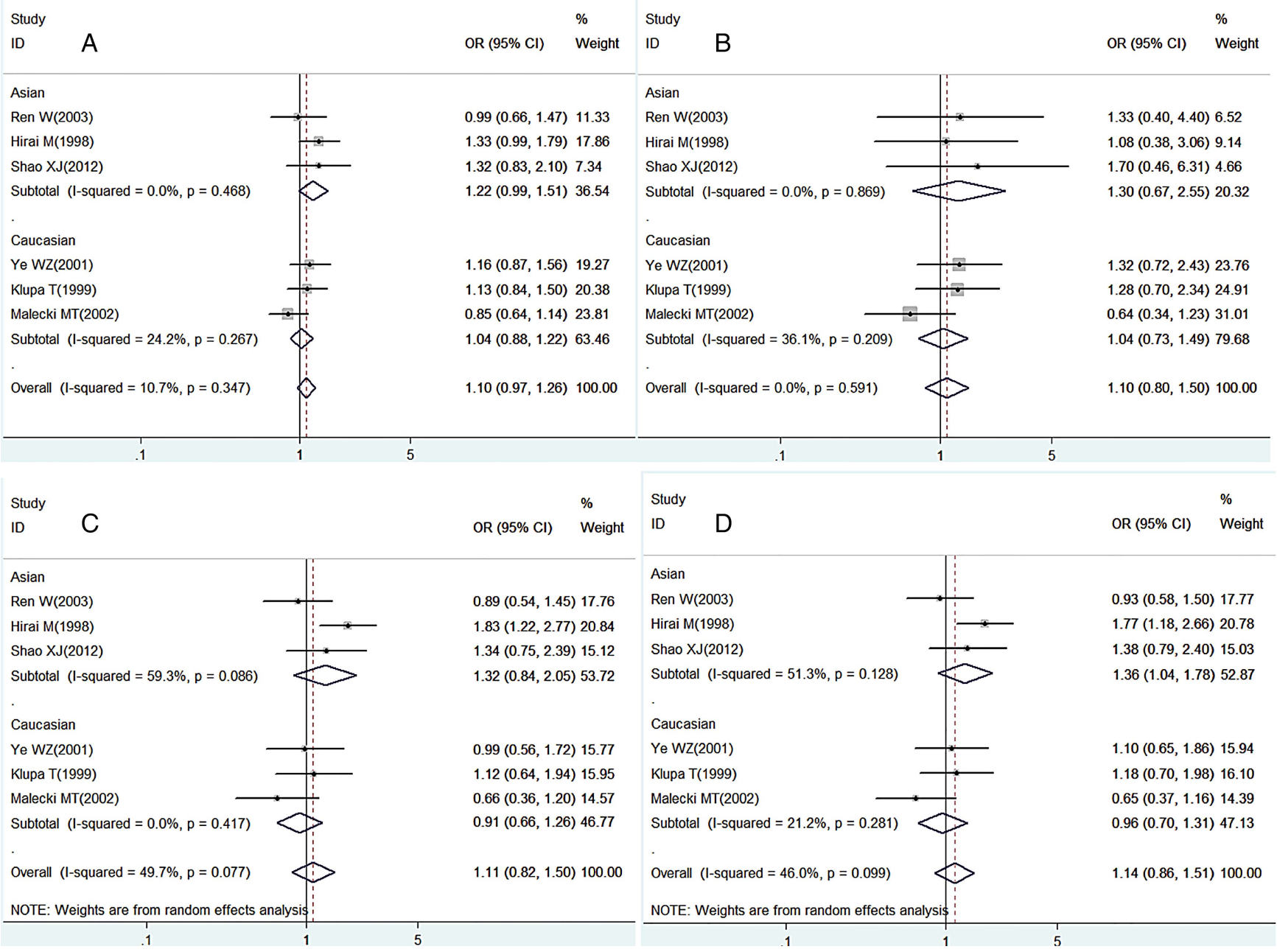

Figure 2 Forest plots describing the association of the codon 416 polymorphism with type 2 diabetes mellitus under (A) Glu versus Asp, (B) Glu/Glu versus Asp/Asp, (C) Glu/Asp versus Asp/Asp and (D) Glu/Asp+Glu/Glu versus Asp/Asp models.

\section{DISCUSSION}

Previous studies have evaluated the association between DBP polymorphisms and the risk of T2DM. ${ }^{11-16}$ However, the results remain conflicting and inconsistent, and thus a systematic review and meta-analysis of association between the DBP polymorphisms and T2DM were of great value. Our findings showed that there was no significant association between codon 420 or codon 416 variation of DBP and T2DM in the overall population as well as in the Caucasian population. However, the polymorphisms of codon 416 and codon 420 in DBP were associated with an increased risk of T2DM in Asians.

The association between DBP andT2DM/prediabetes metabolic traits had been reported in a different population. A study conducted on a Japanese population showed an association between DBP genetic variations and insulin resistance. ${ }^{4}$ Variations in the $D B P$ are associated with oral glucose tolerance in non-diabetic Pima Indians. ${ }^{19}$ Similarly, in Dogrib Indians, the $D B P$ genotype had a significant effect on the fasting insulin level. ${ }^{20}$ A study conducted on the Shanghai population from China also suggested the effect of $D B P$ variations on the function of a $\beta$ cell in a population with abnormal glucose metabolism. ${ }^{21}$ Significant associations between $D B P$ and T2DM were found in the Asian population. $^{111516}$ In Caucasians, however, similar conclusions were not found. ${ }^{12-14}$ So it is possible for us to believe that the effect of variations of $D B P$ on the development of T2DM is peculiar to the Asian population, which is identical to the conclusion of our meta-analysis, in which results of the subgroup analysis showed that individuals carrying the Lys allele or Lys/Thr+Lys/Lys, Lys/ Thr genotypes had more risk for T2DM in the Asian population. Subgroup analysis also suggested that variations at codon 416 were linked with T2DM risk in Asians. These findings might also suggest that T2DM is a disease with heterogeneity in the $D B P$ polymorphisms among populations of different racial, ethnic and geographic backgrounds. In the sensitivity analysis, when the study by Hirai et al was excluded, the evident association became insignificant (before OR (95\% CI) 1.36 (1.04 to 1.78$)$; after OR (95\% CI) 1.10 (0.76 to 1.58$)$ ). So this result should be interpreted cautiously in this population. 


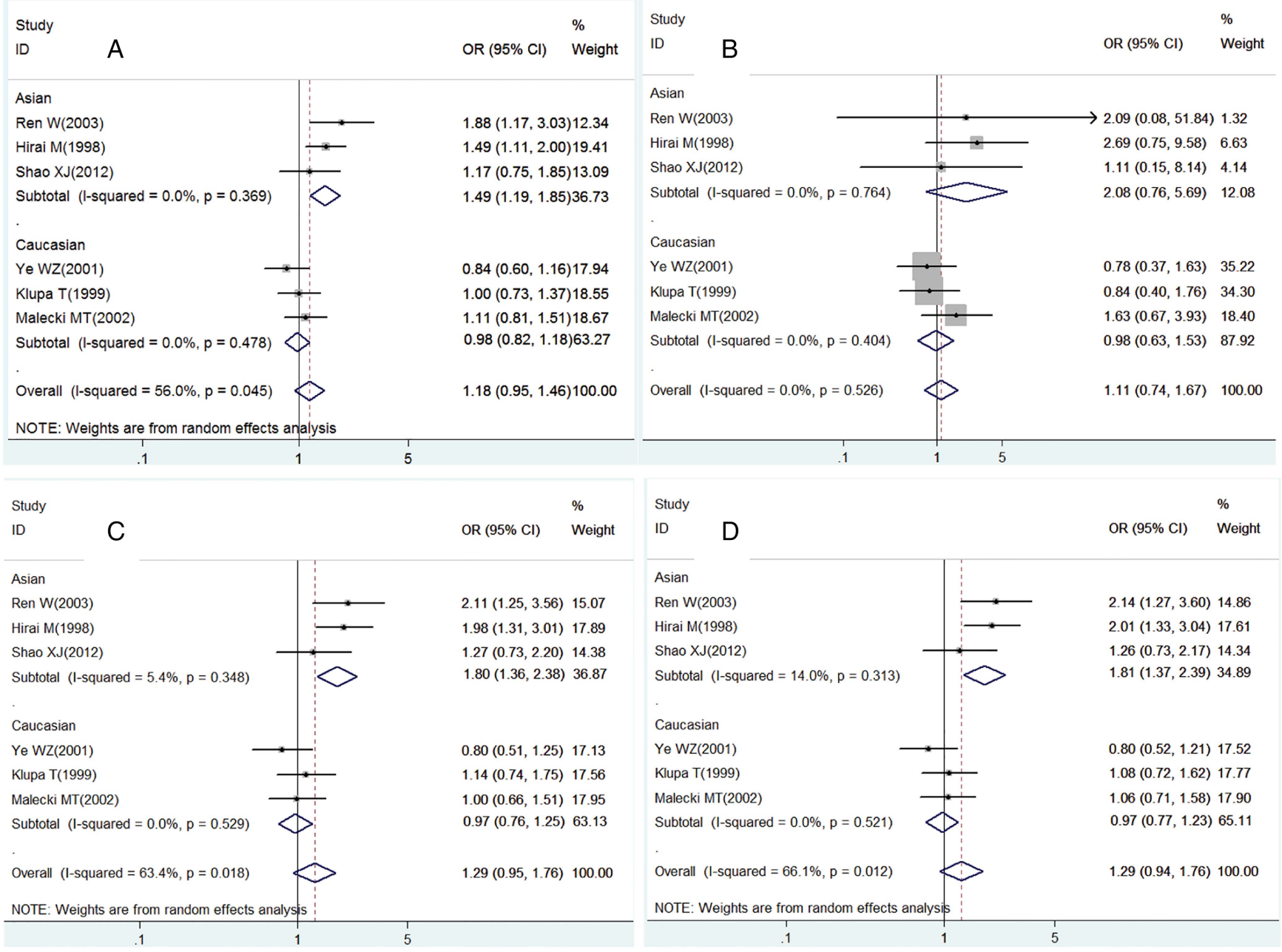

Figure 3 Forest plots describing the association of the codon 420 polymorphism with type 2 diabetes mellitus under (A) Lys versus Thr, (B) Lys/Lys versus Thr/Thr, (C) Lys/Thr versus Thr/Thr and (D) Lys/Thr+Lys/Lys versus Thr/Thr models.

In the present meta-analysis, there existed a significant heterogeneity among the six studies. The results of the exploration of heterogeneity showed that ethnicity was the characteristic leading to heterogeneity under the dominant model in codon 420. Subgroup analysis by ethnicity also proved the conclusion. Stratified analysis based on age or gender, which may be the source of heterogeneity, was not performed on account of lack of information in these studies.
Currently, the mechanism of the association between the $D B P$ polymorphism andT2DM remains unclear. There exist some possible mechanisms. $D B P$ variations have an impact on the metabolite of vitamin $\mathrm{D}$, thereby affecting the amount and activity of vitamin $\mathrm{D}$ in the $\beta$ cell which play an important role in insulin secretion. Another possibility is that the association might be not due to the vitamin D metabolite. Fatty acids, as one of the ligands of DBP, may also induce $\beta$ cell abnormalities

Table 2 Results of meta-regression ( $p$ value)

\begin{tabular}{lllll}
\hline Group & & Case source & Ethnic & Control source \\
\hline Codon 416 & Glu vs Asp & 0.565 & 0.217 & 0.553 \\
& Glu/Glu vs Asp/Asp & 0.468 & 0.522 & 0.725 \\
& Glu/Asp vs Asp/Asp & 0.911 & 0.087 & 0.254 \\
Codon 420 & Glu/Asp+Glu/Glu vs Asp/Asp & 0.839 & 0.090 & 0.286 \\
& Lys vs Thr & 0.316 & 0.051 & 0.600 \\
& Lys/Lys vs Thr/Thr & 0.430 & 0.279 & 0.316 \\
& Lys/Thr vs Thr/Thr & 0.352 & 0.045 & 0.632 \\
\hline
\end{tabular}


Table 3 Pooled ORs and 95\% Cls of overall and subgroup meta-analysis, heterogeneity test and sensitivity analysis in the codon 416 polymorphism

\begin{tabular}{|c|c|c|c|c|c|c|}
\hline \multirow[b]{2}{*}{ Genetic model } & \multicolumn{3}{|l|}{ Meta-analysis } & \multicolumn{3}{|l|}{ Sensitivity analysis } \\
\hline & OR (95\% Cl) & $\mathbf{P h}$ & $\mathrm{I}^{2}(95 \% \mathrm{Cl} ; \%)$ & OR (95\% Cl) & $\mathbf{P h}$ & $\mathrm{I}^{2}(95 \% \mathrm{Cl} ; \%)$ \\
\hline \multicolumn{7}{|l|}{ Codon 416 (overall) } \\
\hline Glu vs Asp & $1.10(0.97$ to 1.26$)$ & 0.35 & $11(0$ to 77$)$ & 1.05 (0.91 to 1.22$)$ & 0.45 & $0(0$ to 79$)$ \\
\hline Glu/Glu vs Asp/Asp & $1.10(0.80$ to 1.50$)$ & 0.59 & $0(0$ to 75$)$ & $1.10(0.79$ to 1.52$)$ & 0.45 & 0 (0 to 79$)$ \\
\hline Glu/Asp vs Asp/Asp & $1.11(0.82$ to 1.50$)$ & 0.08 & $50(0$ to 80$)$ & $0.97(0.76$ to 1.24$)$ & 0.52 & $0(0$ to 79$)$ \\
\hline Glu/Asp+Glu/Glu vs Asp/Asp & $1.14(0.86$ to 1.51$)$ & 0.10 & $46(0$ to 79$)$ & $1.02(0.80$ to 1.29$)$ & 0.42 & 0 (0 to 79$)$ \\
\hline \multicolumn{7}{|c|}{ Codon 416 (Asian) } \\
\hline Glu vs Asp & 1.22 (0.99 to 1.51$)$ & 0.47 & $0(0$ to 90$)$ & 1.12 (0.83 to 1.52$)$ & 0.35 & $0(\mathrm{NA})$ \\
\hline Glu/Glu vs Asp/Asp & $1.30(0.67$ to 2.55$)$ & 0.87 & $0(0$ to 90$)$ & $1.48(0.61$ to 3.60$)$ & 0.79 & 0 (NA) \\
\hline Glu/Asp vs Asp/Asp & $1.32(0.84$ to 2.05$)$ & 0.09 & $59(0$ to 88$)$ & $1.06(0.72$ to 1.54$)$ & 0.29 & 11 (NA) \\
\hline Glu/Asp+Glu/Glu vs Asp/Asp & $1.36(1.04$ to 1.78$)$ & 0.13 & $51(0$ to 86$)$ & $1.10(0.76$ to 1.58$)$ & 0.29 & 11 (NA) \\
\hline \multicolumn{7}{|l|}{ Codon 416 (Caucasian) } \\
\hline Glu vs Asp & 1.04 (0.88 to 1.22$)$ & 0.27 & 24 (0 to 92$)$ & NA & NA & NA \\
\hline Glu/Glu vs Asp/Asp & $1.04(0.73$ to 1.49$)$ & 0.21 & 36 (0 to 80$)$ & NA & NA & NA \\
\hline Glu/Asp vs Asp/Asp & 0.91 (0.66 to 1.26$)$ & 0.42 & $0(0$ to 90$)$ & NA & NA & NA \\
\hline Glu/Asp+Glu/Glu vs Asp/Asp & $0.96(0.70$ to 1.31$)$ & 0.28 & 21 (0 to 92$)$ & NA & NA & NA \\
\hline
\end{tabular}

when it is at a high level in the pancreas islet. ${ }^{19}$ As a macrophage-activating factor, $D B P$ is also critical to the immune system. Several cytokines, such as the tumour necrosis factor, could play important roles in insulin sensitivity. ${ }^{4}$ It is also possible that the association of the $D B P$ polymorphism with T2DM does not result from functional variations in $D B P$, but is derived from a variation in a closely linked gene on chromosome $4 \mathrm{q} 12 .{ }^{11}$

\section{Strengths and limitations}

To the best of our knowledge, this is the first systematic review and meta-analysis to evaluate the association of DBP polymorphisms with T2DM. In this meta-analysis, sensitivity analysis and meta-regression were conducted. In addition, several limitations of this study should also be addressed.
First, the sample size was relatively small for stratified analyses, which weakened our conclusions. Therefore, more studies need to be conducted to obtain a more reliable result. Second, T2DM is a complex metabolic disorder caused by the interaction of multiple genetic and environmental factors; so gene-gene and gene-environment interactions should also be taken into account to conclude a true effect if possible. Third, detailed information at an individual level was lacking in previous studies, so some stratified analyses were not able to be performed. If individual raw data were available, effect induced by age, gender, medication use and other environmental factors (sun exposure, dietary vitamin D intake, etc.) could also be investigated. Fourth, we are unable to control against publication bias for such a small number of studies. Additionally, results of

Table 4 Pooled ORs and 95\% Cls of overall and subgroup meta-analysis, heterogeneity test and sensitivity analysis in the codon 420 polymorphism

\begin{tabular}{|c|c|c|c|c|c|c|}
\hline \multirow[b]{2}{*}{ Genetic model } & \multicolumn{3}{|l|}{ Meta-analysis } & \multicolumn{3}{|l|}{ Sensitivity analysis } \\
\hline & OR (95\% Cl) & $\mathbf{P h}$ & $\mathrm{I}^{2}$ (95\% Cl; \%) & OR (95\% Cl) & $\mathbf{P h}$ & $\mathrm{I}^{2}(95 \% \mathrm{Cl} ; \%)$ \\
\hline \multicolumn{7}{|l|}{ Codon 420 (overall) } \\
\hline Lys vs Thr & $1.18(0.95$ to 1.46$)$ & 0.05 & 56 (0 to 82$)$ & $1.11(0.88$ to 1.40$)$ & 0.10 & $49(0$ to 81$)$ \\
\hline Lys/Lys vs Thr/Thr & $1.11(0.74$ to 1.67$)$ & 0.53 & 0 (0 to 75$)$ & $1.00(0.65$ to 1.54$)$ & 0.73 & 0 (0 to 79$)$ \\
\hline Lys/Thr vs Thr/Thr & $1.29(0.95$ to 1.76$)$ & 0.02 & 63 (11 to 85$)$ & $1.17(0.86$ to 1.58$)$ & 0.08 & $52(0$ to 82$)$ \\
\hline $\begin{array}{l}\text { Lys/Thr+Lys/Lys vs Thr/Thr } \\
\text { Codon } 420 \text { (Asian) }\end{array}$ & 1.29 (0.94 to 1.76$)$ & 0.01 & 66 (19 to 86$)$ & 1.16 (0.86 to 1.57$)$ & 0.07 & 54 (0 to 83) \\
\hline Lys vs Thr & $1.49(1.19$ to 1.85$)$ & 0.37 & $0(0$ to 90$)$ & 1.48 (1.07 to 2.05$)$ & 0.16 & 50 (NA) \\
\hline Lys/Lys vs Thr/Thr & $2.08(0.76$ to 5.69$)$ & 0.76 & 0 (0 to 90$)$ & $1.35(0.25$ to 7.12$)$ & 0.74 & 0 (NA) \\
\hline Lys/Thr vs Thr/Thr & $1.80(1.36$ to 2.38$)$ & 0.35 & 5 (0 to 90$)$ & $1.67(1.14$ to 2.43$)$ & 0.19 & 42 (NA) \\
\hline $\begin{array}{l}\text { Lys/Thr+Lys/Lys vs Thr/Thr } \\
\text { Codon } 420 \text { (Caucasian) }\end{array}$ & 1.81 (1.37 to 2.39 ) & 0.31 & $14(0$ to 91$)$ & 1.67 (1.15 to 2.43 ) & 0.17 & 47 (NA) \\
\hline Lys vs Thr & $0.98(0.82$ to 1.18$)$ & 0.48 & 0 (0 to 90$)$ & NA & NA & NA \\
\hline Lys/Lys vs Thr/Thr & $0.98(0.63$ to 1.53$)$ & 0.40 & 0 (0 to 90$)$ & NA & NA & NA \\
\hline Lys/Thr vs Thr/Thr & $0.97(0.76$ to 1.25$)$ & 0.53 & $0(0$ to 90$)$ & NA & NA & NA \\
\hline Lys/Thr+Lys/Lys vs Thr/Thr & $0.97(0.77$ to 1.23$)$ & 0.52 & 0 (0 to 90$)$ & NA & NA & NA \\
\hline
\end{tabular}


Genome-wide association study (GWAS) studies were not included because the raw data had not been published.

\section{CONCLUSIONS}

In conclusion, this meta-analysis had pooled all the available data related to the $D B P$ polymorphism and T2DM, and indicated that the $D B P$ polymorphism was only moderately associated with an increased susceptibility to T2DM in Asians but not in Caucasians. Therefore, more well-designed and large sample studies are warranted to confirm this conclusion, and to fully understand the mechanism of T2DM. Additionally, prospective cohort studies in combination with analyses of other gene and environment factors are also necessary to explore the true effect of the $D B P$ polymorphism on the risk of T2DM.

Acknowledgements The authors would like to thank Jiejie Qin, MSc, and Shangshu Zhang, MSc, for helping with data analysis during the research. The authors also wish to thank Dr Mark Dickson and Dr Fatiha Karam for their critical reading of the manuscript.

Contributors CW, WL, GW and YL contributed to the conception and design, acquisition of the data, analysis and interpretation of the data, and the drafting of the articles. LL, FY, LC and YB were involved in the collection and analysis of the data. All authors approved the final version of the manuscript.

Funding This work was supported by the National Natural Science Foundation of China (Grant no: U1204823 and U1304821), National Key Basic Research Program of China (Grant no: 2012CB526709), High-level Personnel Special Support Project of Zhengzhou University (no: ZDGD13001), China Postdoctoral Science Foundation (Grant no: 20100471003 and 201104401) and Medical Scientific Research Foundation of Health Department of Henan Province (Grant no: 201004042 and 201204051).

Competing interests None.

Ethics approval Ethics approval was granted by the Zhengzhou University Medical Ethics Committee.

Provenance and peer review Not commissioned; externally peer reviewed.

Data sharing statement No additional data are available.

Open Access This is an Open Access article distributed in accordance with the Creative Commons Attribution Non Commercial (CC BY-NC 4.0) license, which permits others to distribute, remix, adapt, build upon this work noncommercially, and license their derivative works on different terms, provided the original work is properly cited and the use is non-commercial. See: http:// creativecommons.org/licenses/by-nc/4.0/

\section{REFERENCES}

1. Talmud PJ, Hingorani AD, Cooper JA, et al. Utility of genetic and non-genetic risk factors in prediction of type 2 diabetes: Whitehall II prospective cohort study. BMJ 2010;340:b4838.
2. $\mathrm{Li} \mathrm{H}, \mathrm{Gan} \mathrm{W}$, Lu L, et al. A genome-wide association study identifies GRK5 and RASGRP1 as type 2 diabetes loci in Chinese Hans. Diabetes 2013;62:291-8.

3. Wang CJ, Li LL, Wang L, et al. Evaluating the risk of type 2 diabetes mellitus using artificial neural network: an effective classification approach. Diabetes Res Clin Pract 2013;100:111-18.

4. Hirai M, Suzuki S, Hinokio $Y$, et al. Variations in vitamin D-binding protein (group-specific component protein) are associated with fasting plasma insulin levels in Japanese with normal glucose tolerance. J Clin Endocrinol Metab 2000;85:1951-3.

5. Braun A, Kofler A, Morawietz S, et al. Sequence and organization of the human vitamin D-binding protein gene. Biochim Biophys Acta 1993;1216:385-94.

6. Afzal S, Bojesen SE, Nordestgaard BG. Low 25-hydroxyvitamin D and risk of type 2 diabetes: a prospective cohort study and meta-analysis. Clin Chem 2012;59:381-91.

7. Flores $M$. A role of vitamin $D$ in low-intensity chronic inflammation and insulin resistance in type 2 diabetes mellitus? Nutr Res Rev 2005;18:175-82.

8. Chiu KC, Chu A, Go VL, et al. Hypovitaminosis D is associated with insulin resistance and beta cell dysfunction. Am J Clin Nutr 2004;79:820-5.

9. Vu D, Sakharkar P, Tellez-Corrales E, et al. Association of vitamin D binding protein polymorphism with long-term kidney allograft survival in Hispanic kidney transplant recipients. Mol Biol Rep 2013;40:933-9.

10. Ongagna JC, Kaltenbacher MC, Sapin R, et al. The HLA-DQB alleles and amino acid variants of the vitamin $\mathrm{D}$ binding protein in diabetic patients in Alsace. Clin Biochem 2001;34:59-63.

11. Hirai M, Suzuki S, Hinokio Y, et al. Group specific component protein genotype is associated with NIDDM in Japan. Diabetologia 1998;41:742-3.

12. Klupa T, Malecki M, Hanna L, et al. Amino acid variants of the vitamin D-binding protein and risk of diabetes in white Americans of European origin. Eur J Endocrinol 1999;141:490-3.

13. Ye WZ, Dubois-Laforgue D, Bellanné-Chantelot $\mathrm{C}$, et al. Variations in the vitamin D-binding protein (Gc locus) and risk of type 2 diabetes mellitus in French Caucasians. Metabolism 2001;50:366-9.

14. Malecki MT, Klupa T, Wanic K, et al. Vitamin D binding protein gene and genetic susceptibility to type 2 diabetes mellitus in a polish population. Diabetes Res Clin Prac 2002;57:99-104.

15. Ren W, Zhang SH, Wu J, et al. Research of amino acid variants of the vitamin $\mathrm{D}$ binding globulin in type 2 diabetes family. Chin J Prev Med 2003;37:202-3.

16. Shao XJ, Miao H, Jiang XQ, et al. Association of vitamin D binding protein receptor gene polymorphism and risk of type 2 diabetes mellitus in Han people of Nanjing Province. Chin J Mod Med 2012;22:45-8

17. Moher D, Liberati A, Tetzlaff $\mathrm{J}$, et al. Preferred reporting items for systematic reviews and meta-analyses: the PRISMA statement PLoS Med 2009;6:e1000097.

18. Wells G, Shea B, O'Connell D, et al. NewCastle-Ottawa Quality Assessment Scale-Case Control Studies. http://www.ohri.ca/ programs/clinical_epidemiology/oxford.asp (accessed 20 Jun 2014).

19. Baier LJ, Dobberfuhl AM, Pratley RE, et al. Variations in the vitamin $D$ binding protein (Gc locus) are associated with oral glucose tolerance in nondiabetic Pima Indians. J Clin Endocrinol Metab 1998:83:2993-6.

20. Szathmary EJ. The effect of GC genotype on fasting insulin level in Dogrib Indians. Hum Genet 1987:75:368-72.

21. Wang YB, Yu YC, Li Z, et al. Correlation between polymorphism of vitamin $D$ binding protein gene and type 2 diabetes mellitus in Han people of Shanghai. Chin J Endocrinol Metab 2006;22:263-4. 